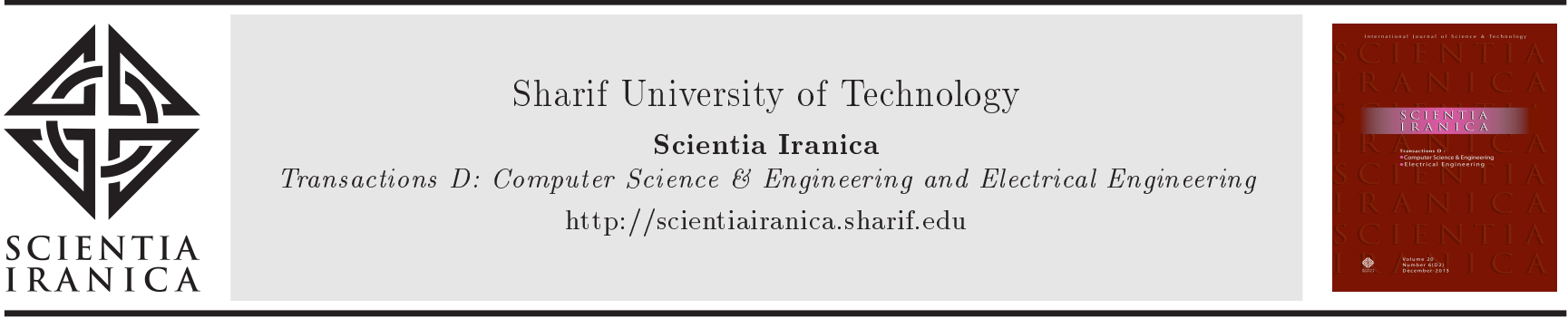

\title{
A quick solution to optimal coordinated voltage control based on dimension-reduction of power system through modified Ward-PV
}

\author{
M. Hojati Tabatabaei, H. Siahkali*, and J. Olamaei \\ Department of Electricity Engineering, South Tehran Branch, Islamic Azad University, Mahallati Exp., Tehran, P.O. Box \\ 177r7613651, Iran.
}

Received 14 October 2020; received in revised form 10 March 2021; accepted 2 May 2021

\author{
KEYWORDS \\ Voltage stability; \\ Modified Ward-PV; \\ OCVC; \\ Reduction of the \\ system dimensions.
}

\begin{abstract}
Voltage control and voltage stability are the two most important issues in power systems. Accordingly, the current study primarily aims to investigate the emergency voltage control in power systems. In this regard, one of the most important methods proposed to solve this problem is Optimal Coordinated Voltage Control (OCVC), which operates based on Model Predictive Control (MPC). In order to solve an OCVC problem, time limitations are taken into account to prevent voltage collapse as the most critical constraint. In this research, modified Ward-PV was proposed to reduce the dimensions of the power system and consequently solve the problem faster. In this method, immediately after a fault occurrence, the power system is partitioned into three subsystems based on spectral graph partitioning method. Partitioning in this method is done based on the reactive power flow through the lines. The subsystems, which are far from fault, will be replaced by the reduced model. This method would in turn drastically reduce the number of system equations and boost the process of problem-solving. The results of simulations obtained from New England IEEE 39-Bus System revealed that the function of the proposed method was more precise than that of the dimension reduction method of the power system based on linearization of external subsystems. However, it impeded the process of problem-solving. (C) 2022 Sharif University of Technology. All rights reserved.
\end{abstract}

\section{Introduction}

Voltage stability is one of the main concerns in the power systems. Based on IEEE/CIGRE definition, voltage stability is the ability of the power system to maintain an acceptable steady state voltage for all buses after a disturbance $[1,2]$.

Voltage collapse is a severe voltage drop of a

*. Corresponding author. Tel.: +982133722831 E-mail addresses: m.h.tabatabaiee2007@gmail.com ( $M$. Hojati Tabatabaei); h_siahkali@azad.ac.ir (H. Siahkali); J_olamaei@azad.ac.i (J. Olamaei)

doi: $10.24200 /$ sci. 2021.56970 .4999 power system resulting from voltage instability. In case some buses undergo voltage drop, generators or other reactive power compensators will compensate it and the voltage status will remain in its normal mode. On the contrary, if the system faces another problem, the voltage drop may be more severe and the undervoltage relays cause outage of some generators. As a result, the voltage drop in the system becomes more severe and a blackout occurs finally. A comprehensive study of blackouts all around the world confirms that voltage drop is the main cause of most blackouts $[3,4]$. In addition to considerable economic damages, these blackouts will also cause dissatisfaction among people with the electricity network. This is the main reason why rapid identification of unstable conditions and 
appropriate control actions play a significant role in increasing the quality and security of the system.

There are two ways of voltage control in a power system called the preventive and emergency controls. The set of actions taken prior to fault occurrence to improve profile voltage is called preventive control, and the set of control actions taken followed by fault occurrence in the power system to prevent voltage collapse is called emergency control. The present study aims to explore the emergency voltage control where time shortage as the most important factor is of significance. In case the required control actions are not applied to the power system at the right time, the system will be out of its absorption area and subsequently, voltage collapse will definitely occur [5-8].

To prevent voltage collapse in a power system, an optimal coordinated and proper arrangement of control actions called Optimal Coordinated Voltage Control (OCVC) can be employed [9]. Nowadays, Coordinated Voltage Control (CVC) is successfully implemented in France, Italy, Belgium, and Switzerland [10-14]. However, it should be noted that CVC requires more research in large power systems.

Figure 1 represents the structure of all previous studies on the emergency voltage control. Some papers have addressed the issue in multi-area power systems where each area is exclusively devoted to its own operator with no access to any information from other areas. The Lagrangian decomposition was proposed to solve the emergency voltage control problem in multi-area power systems [15-22]. Studies on the emergency voltage control in a single-area system have assumed that the power system operator is aware of all system parameters at any time which can use all

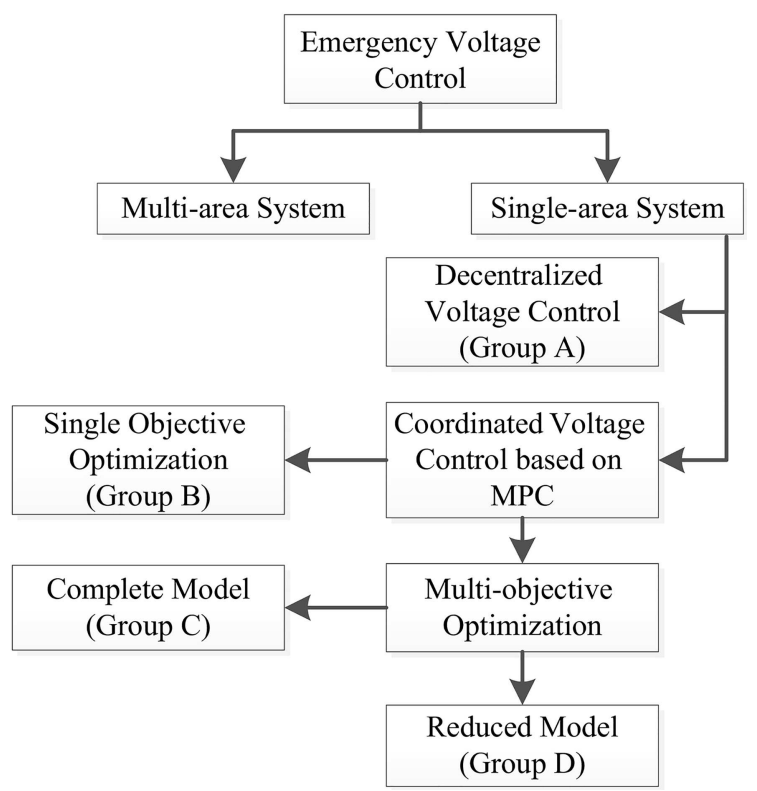

Figure 1. Classification of studies on the emergency voltage control. available control actions to control the system [2347]. The current study aims to investigate the problem of emergency voltage control in a single-area system, assuming that all system parameters, such as voltage, current, fault location, etc., are available.

Generally, research on the single-area power systems can be categorized into two main groups: (1) decentralized voltage control [42-45] and (2) CVC based on Model Predictive Control (MPC) method [2341,46]. These two categories are also divided into four Groups A to D in Figure 1.

The power system equations in the emergency voltage control problem are differential-algebraic, and the nature of the control variables is a combination of discrete and continuous variables. Therefore, it can be concluded that the OCVC is an optimization problem with a high degree of complexity. In this regard, the need for a proper and fast control system in the OCVC problem is felt more than ever. Features of a good and complete method for solving the emergency voltage control problem are listed in the following:

1. It should consider all equality and inequality constraints;

2. It should be an adaptive method to ensure its adaptability by changing the operating point;

3. It should not use load shedding to control the system as far as possible;

4. It should be an optimal method. In other words, the optimal control actions should be applied when needed;

5. It should have appropriate flexibility, meaning that the operator is given the opportunity to choose the optimal solution from the set of non-dominated solutions obtained from solving a multi-objective optimization problem;

6. It should perform well against any unanticipated fault, i.e., any disturbance except the outage of a line or a generator;

7. It should perform well against consecutive faults;

8. It should be able to determine the optimal solution at an appropriate speed;

9. It should be applicable to large power systems.

Based on these nine features, Table 1 makes a comparison of the studies classified into Groups A to D.

In the decentralized control strategy, each distributed energy source operates freely using the measured local signals $[7,48]$. In the papers of Group A under the title of "Decentralized Voltage Control", the power system is initially partitioned into several subsystems and one control system and one performance index are defined for each subsystem. Whenever the performance index of one subsystem is greater than 1 , 
Table 1. Comparison of the studies of Groups A to D.

\begin{tabular}{lcccc}
\hline \multicolumn{1}{c}{ Description } & Group A & Group B & Group C & Group D \\
\hline Consider all constraints & $\sqrt{ }$ & $\sqrt{ }$ & $\sqrt{ }$ & $\sqrt{ }$ \\
Adaptive method & $\sqrt{ }$ & $\sqrt{ }$ & $\sqrt{ }$ & $\sqrt{ }$ \\
Optimal method & $\times$ & $\sqrt{ }$ & $\sqrt{ }$ & $\sqrt{ }$ \\
Minimum load shedding & $\times$ & $\times \sqrt{ }(1)$ & $\sqrt{ }$ & $\sqrt{ }$ \\
Flexibility method & $\times$ & $\times$ & $\sqrt{ }$ & $\sqrt{ }$ \\
Good performance against unanticipated fault & $\sqrt{ }$ & $\times$ & $\times$ & $\sqrt{ }$ \\
Good performance against consecutive faults & $\sqrt{ }$ & $\sqrt{ }$ & $\sqrt{ }$ & $\sqrt{ }$ \\
Speed of solving problem & High & Low & Low & High \\
Applied in large power system & $\sqrt{ }$ & $\times$ & $\times$ & $\sqrt{ }$ \\
\hline
\end{tabular}

(1) This depends on the objective function (Tracing reference voltage / Minimizing number of control actions /

Minimizing load shedding).

the control actions of that subsystem will be implemented continuously with an interval of five seconds. Fast recovery of the voltage is an advantage of this method. However, it has also some disadvantages such as lack of optimal control actions and large number of control actions which make the control system more costly [42-45].

In the papers of Groups $\mathrm{B}$ and $\mathrm{C}$, the emergency voltage control method is based on MPC which is similar to Proportional Integral Derivative (PID) control methods, except that in the PID-based controllers, control measures are determined according to the feedback received from the output and applied to the system, while in the MPC method, the behavior of the power system becomes predictable in the future by applying a set of control actions to the system at the moment [33]. $\mathrm{MPC}$ is used to control different problems in the power system $[49,50]$. In the MPC-based coordinated voltage control, optimal control measures are determined by solving either single- or multi-objective optimization problems. The objective functions generally include tracing the reference voltage, reducing the number of control actions, and minimizing load shedding. In Group B, tree search, evolutionary algorithm, heuristic search, pseudo-gradient, and Particle Swarm Optimization (PSO) algorithm are generally used to solve the single-objective problems [23-35]. The main advantage of the papers in Group B is their capability of finding optimal control actions; however, the proposed method does not have the flexibility feature given its ability to solve the single-objective optimization. In order to overcome this disadvantage, papers in Group $\mathrm{C}$ that solved the multi-objective optimization are suggested [36-40,46]. By solving the multi-objective optimization, a set of non-dominated solutions can be obtained; hence, the system operator is given the opportunity to choose the best control actions from the operational, economic, or environmental points of view and apply it to the system according to the system conditions. In this case, it is supposed that the control system is characterized by the flexibility feature.

Finding solutions to methods of optimization problems are generally iterative and time-consuming. In this regard, several calculations were done in online and offline parts to boost the speed of the control systems [36-40]. Offline calculations provide an appropriate knowledge base, which can also be used for online part. Nevertheless, the speed of the control system is not suitable for solving the emergency voltage control in large power systems. In this respect, the papers of Group D are suggested to solve this problem. In [41], the similar method to [36-40] was employed to solve the OCVC problem, with one large difference in increasing the speed, arguing that voltage instability at early moments was a local phenomenon; hence, the areas far from the fault location were replaced by the dimension reduction method based on linearization. Consequently, the number of equations of the system decreased and the speed of control method increased. Through linearization of the external subsystems, their equations and external control variables were linearized, which considerably lessened the accuracy of solving the optimization problem.

Interested in the nonlinearity and complexity of the power system, operators and scientists in the field of electricity industry have constantly attempted to find a way to reduce power system dimensions and calculation time. Ward and Ward-PV methods are among the efficient methods used for reducing the dimensions of the power system [51]. The superiority of the Ward$\mathrm{PV}$ method over the Ward method is its preservation of $\mathrm{PV}$ buses in the external subsystems in controlling the system. It can be predicted that compared to the linearization method, due to the greater numbers of equations, calculations in this method may be notably time consuming. 
Given that the susceptance of capacitors was used for OCVC in this research, the Ward-PV method was modified; besides PV buses, capacitor buses were also preserved in the external areas. The main innovations of this study are summarized in the following:

1. Owing to its ability to solve the multi-objective optimization problem (which will be fully described later), the proposed method is characterized by the abovementioned features 1 to 7 . In addition, given that the proposed method functions based on reducing the system dimensions using the modified Ward-PV, features 8 and 9 should also be included. Therefore, the proposed method is a comprehensive one that can be employed to solve the emergency voltage control problem, which will be proven based on three simulation scenarios;

2. The spectral graph partitioning method is used for partitioning the power system followed by fault occurrence. Appropriate system partitioning should be done under two conditions: (a) the effect of fault on the voltage of external buses should be less than that on the voltage of the internal ones and (b) followed by fault occurrence, buses that are greatly affecting the restoration of fault zone voltages should be placed in the fault zone. In [3945,51-53], the system was partitioned before the fault occurrence; therefore, partitioning was done regardless of these two conditions;

3. Due to the correlation between the reactive power and voltage, partitioning is done based on the reactive power flow. In [41], partitioning was done based on the admittance matrix of the system. However, in case the occurring fault is a generator outage or a change in the system load, the admittance matrix of the system will not change. Hence, it can be concluded that partitioning based on admittance matrix cannot be precise;

4. The power system dimensions can be reduced using the modified Ward-PV method. One of the advantages of this method is retaining effective control variables in recovering the voltage of subsystem, being far from the fault location. In addition, it is more precise than the linearization method introduced in [41].

The structure of the paper is as follows: Section 2 elaborates the power system model in the emergency voltage control problem. Section 3 discusses the OCVC, objective functions, and Multi-Criteria Decision-Making (MCDM). Section 4 explains how to use the modified Ward-PV technique for reducing the dimensions of the power System. Section 5 thoroughly examines the proposed algorithm of the problem. Section 6 presents the simulation attempts under three scenarios. Finally, Section 7 presents the obtained results and concludes the study.

\section{Model of power system in emergency voltage control}

The power system model in the emergency voltage control is composed of a set of differential-algebraic equations, expressed as Eqs. (1) and (2):

$$
\begin{aligned}
& \dot{x}=f(t, x, y, u), \\
& 0=g(x, y, u),
\end{aligned}
$$

where $x, y$, and $u$ are the state variables, algebraic variables, and control actions, respectively. Since we intend to study long-term voltage instability problems, the quasi-steady-state approximation of generators would be sufficient to study the essential behavior of the system. Hence, the differential equations of the system are the only the differential equations of the loads. The aggregate exponential recovery load model was employed in this paper and the relative equations are given below $[33,41,42]$ :

$$
\begin{aligned}
& d x_{i, p} / d t=-x_{i, p} / T_{i, p}+P_{i, 0}\left(V_{i}^{\alpha_{s}}-V_{i}^{\alpha_{t}}\right), \\
& P_{i, d}=\left(1-n_{i, d} D_{\text {shed }}\right)\left(x_{i, p} / T_{i, p}+P_{i, 0} V_{i}^{\alpha_{t}}\right), \\
& d x_{i, q} / d t=-x_{i, q} / T_{i, q}+Q_{i, 0}\left(V_{i}^{\beta_{s}}-V_{i}^{\beta_{t}}\right), \\
& Q_{i, d}=\left(1-n_{i, d} D_{\text {shed }}\right)\left(x_{i, q} / T_{i, q}+Q_{i, 0} V_{i}^{\beta_{t}}\right),
\end{aligned}
$$

where $V_{i}$ is the voltage of bus $i$, and $x_{i, p}$ and $x_{i, q}$ are the state variables that when changed, the active and reactive power in the $i$ th bus will be recovered. For this reason, when the voltage decreases, the values of $x_{i, p}$ and $x_{i, q}$ begin to increase to recover the power. In addition, $P_{i, d}$ and $Q_{i, d}$ are the active and reactive power of the load at bus $i$, respectively. Moreover, $D_{\text {shed }}$ represents the load shedding step equal to $0.05 \mathrm{pu}$, and $n_{i, d}$ is the number of load shedding steps at the bus $i . T_{i, p}$ and $T_{i, q}$ are the time constants of the active and reactive power loads, respectively. $T_{i, p}$ and $T_{i, q}$ change from several ten seconds to several minutes depending on the recovery factor. These values in this research were considered to be 60 seconds. Of note, $\alpha_{s}$, $\alpha_{t}, \beta_{s}$, and $\beta_{t}$ are the constant factors indicating the degree of the dependence of active and reactive power of loads on the steady and transient states. It should be noted that $\alpha_{t}$ and $\beta_{t}$ are always greater than $\alpha_{s}$ and $\beta_{s}$, meaning that the degree of the dependence of load on the voltage in the transient state is greater than that in the steady state. The control parameters in this study include the susceptance of capacitors, reference voltage of each generator, On-Load Tap Changers (OLTC), and load shedding. 


\section{Optimal Coordinated Voltage Control (OCVC)}

The concept of CVC refers to the coordinated use of resources to control reactive power of the system simultaneously. It is proved that coordinated use of control equipment in different locations instead of using big control equipment in one place is more efficient in improving the system condition. For the first time, the MPC was proposed to solve the CVC problem in [33]. This research employed the MPC to solve the OCVC problem. Figure 2 demonstrates the structure of OCVC based on MPC according to which the control variables $u$ are selected in terms of future requirements and applied to the power system. To this end, the variables of the system, i.e., $x$ and $y$, were first sampled. Then, based on sampled values, the output of the system $\hat{y}$ was predicted for each arrangement of control actions $u$. Subsequently, the control actions of outputs that are closer to the reference value $y r$ will be applied to the system. In OCVC, $\hat{y}$ is the voltage of the buses. Euler method was employed to predict the response path $\hat{y}$ [33]. The OCVC problem is considered as a multi-objective optimization problem as in [36-41]. Followed by solving the multi-objective optimization, a set of non-dominated solutions is obtained. Therefore, the system operator is provided with the opportunity to choose the best control actions from the operational, economic, or environmental points of view and apply them to the system according to the system conditions using the MCDM technique. These three objective functions are as follows:

$$
\begin{aligned}
& J_{\sum V i}=\min \sum_{i} \int_{t_{0}}^{t_{p}}\left|v_{i t}-v_{\text {iref }}\right| d t \\
& J_{\text {act }}=\min n_{c} \\
& J_{\text {load }}=\min \sum_{k} n_{i, d},
\end{aligned}
$$

where $J_{\sum V i}, J_{a c t}$, and $J_{\text {load }}$ represent the tracing reference voltage, number of control actions, and load

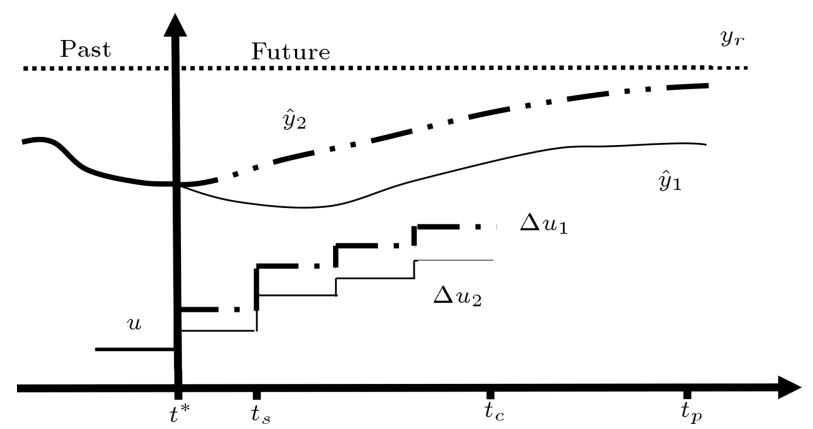

Figure 2. The control structure of Optimal Coordinated Voltage Control (OCVC) based on Model Predictive Control (MPC). shedding, respectively. In addition, $v_{i t}$ is the voltage of bus $i$ at time $t$ and $v_{\text {iref }}$ is the reference voltage of bus $i$, which is assumed to be $1 \mathrm{pu}$. Moreover, $t_{p}$ and $t_{0}$ are the prediction interval and moment of applying the control actions, respectively. Further, $n_{c}$ represents the number of control actions applied to the system and $n_{i, d}$ is the number of load shedding steps in bus $i$. Similar to [36-41], jumping genes paradigm optimization algorithm was proposed in this study to solve the multi-objective optimization problem.

\subsection{Multi-Criteria Decision-Making (MCDM)}

Followed by finding a set of non-dominated solutions, the system operator uses the MCDM technique to determine the best answer and apply it on the system. This technique is completely explained in [37-40]. Based on three objective functions and $n$ optimal nondominated solutions, the solutions are ranked according to Eq. (10) and finally, the first-rank solution will be applied to the system:

$$
r_{i}=\sum_{i=1}^{3} w_{j} a_{i j n},
$$

where $r_{1}, \ldots, r_{n}$ are the final ranks of the non-dominated solutions $A_{1}, \ldots, A_{n}$, respectively (see Table 2 ). In addition, $w_{1}, w_{2}$, and $w_{3}$ denote the relative importance of $J_{\sum V i}, J_{\text {act }}$, and $J_{\text {load }}$ obtained from Eq. (12). Of note, $a_{i j n}$ is the normalized value $a_{i j}$ which is calculated through Eq. (11):

$$
a_{i j n}=\frac{\Gamma\left(a_{i j}-a_{w j}\right)}{\left(a_{b j}-a_{w j}\right)}+1 .
$$

If we assume that $\Gamma=99, a_{i j n}$ values fall into the $\operatorname{span}[1,100]$. In the above equation, $a_{b j}$ and $a_{w j}$ are the best and worst values of the objective function $J_{j}$, respectively. To determine $w_{j}$, first, we must form a preference matrix given in Table 3 in which $m_{i i}=1$ and $m_{i j}=1 / m_{j i}$. The expression $m_{i j}$ denotes that $J_{i}$ possesses a value $m_{i j}$ times $J_{i}$. For example, if the value of $m_{23}$ is assumed to be 3 , the importance of the objective function $J_{2}$ is three times that of the objective function $J_{3}$. Therefore, we have:

$$
M w=\lambda_{\max } w,
$$

where $\lambda_{\max }$ is the maximum eigenvalue of the preference matrix $M$ and $w$ the eigenvector of this eigenvalue.

Table 2. Set of non-dominated solutions.

\begin{tabular}{cccc}
\hline & $\boldsymbol{f}_{\mathbf{1}}=\boldsymbol{J}_{\sum \boldsymbol{v i}}$ & $\boldsymbol{f}_{\mathbf{2}}=\boldsymbol{J}_{\text {act }}$ & $\boldsymbol{f}_{\mathbf{3}}=\boldsymbol{J}_{\text {local }}$ \\
\hline$A_{1}$ & $a_{11}$ & $a_{12}$ & $a_{13}$ \\
$A_{2}$ & $a_{21}$ & $a_{22}$ & $a_{23}$ \\
$\ldots$ & $\ldots$ & $\ldots$ & $\ldots$ \\
$A_{i}$ & $a_{i 1}$ & $a_{i 2}$ & $a_{i 3}$ \\
$\ldots$ & $\ldots$ & $\ldots$ & $\ldots$ \\
$A_{n}$ & $a_{n 1}$ & $a_{n 2}$ & $a_{n 3}$ \\
\hline
\end{tabular}


Table 3. Preference matrix of tracing reference voltage, number of control actions, and load shedding.

\begin{tabular}{cccc}
\hline & $\boldsymbol{f}_{\mathbf{1}}=\boldsymbol{J}_{\sum \boldsymbol{v i}}$ & $\boldsymbol{f}_{\mathbf{2}}=\boldsymbol{J}_{\text {act }}$ & $\boldsymbol{f}_{\mathbf{3}}=\boldsymbol{J}_{\text {local }}$ \\
\hline$f_{1}=J_{\sum v i}$ & $m_{11}$ & $m_{12}$ & $m_{13}$ \\
$f_{2}=J_{\text {act }}$ & $m_{21}$ & $m_{22}$ & $m_{23}$ \\
$f_{3}=J_{\text {local }}$ & $m_{31}$ & $m_{32}$ & $m_{33}$ \\
\hline
\end{tabular}

\section{Reducing the system dimensions}

Modern power systems are interconnected to provide acceptable levels of reliability. Each Energy Management System (EMS) is only appropriate for a small part of this interconnected system called the internal zone. The rest of the system, which is not monitored by EMS, is called the external zone. To effectively monitor the internal zone and evaluate its security, it is necessary to select a suitable model for the external zone which can properly show the impact of the external zone on the internal one. In studies such as those on the emergency voltage control, in case the external zone is modeled in great detail, the degree of the problem complexity will be high. For this reason, the reduced models are used for the external zones. Different methods are employed for reducing the dimension of the system. For example, Ward and Ward-PV are two static methods for reducing the dimensions of the power system, as shown in Figure 3(a) and (b). Given that the voltage instability phenomenon is only a local issue at the early stages of fault occurrence in the power system [45], Ward and Ward-PV can be used in zones that are far from fault. Compared to the method presented in [41], the Ward-PV method used in this paper was modified. All external buses in the Ward method were removed from the system and other buses except for the PV buses of external zones were removed from the model in the Ward-PV method. On the contrary, capacitor buses as well as PV ones remain in the system in the proposed modified Ward-PV method. Figure 3(c) shows the modified Ward-PV model.

In order to reduce the dimensions, the power system should be partitioned immediately after the fault occurrence. A power system can be assumed as a graph with buses comprising a set of vertexes, and the line between the two buses is the branch of the graph [54]. The amount of each branch equals that of the reactive power flow from the line immediately after fault occurrence. Consequently, the power system is partitioned in a way that the sum of the amounts of the reactive power flow between branches of sub-areas be minimized. In other words, areas can be independent in supplying the needed reactive power and preserving their voltages as much as possible.

Followed by partitioning the power system and determining the external areas that are far from the fault, the modified Ward-PV will be implemented on

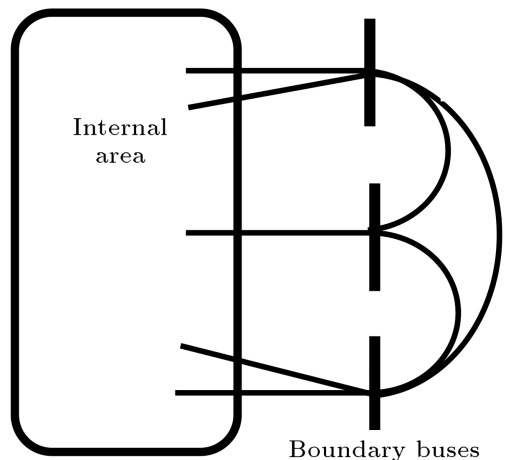

(a)

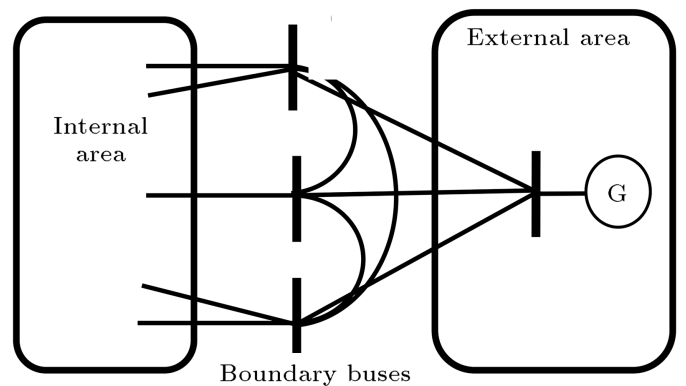

(b)

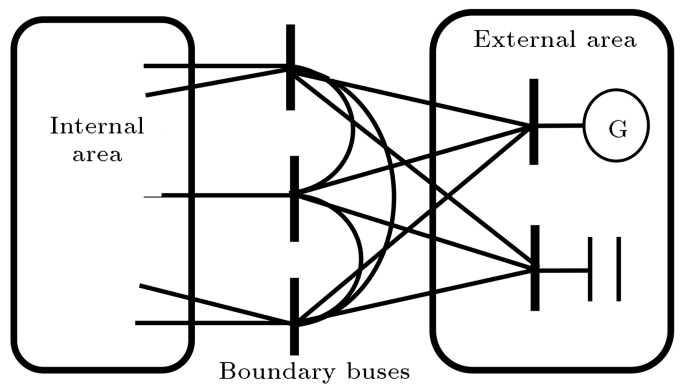

(c)

Figure 3. (a) Ward, (b) Ward-PV, and (c) modified Ward-PV models.

the system. The modified Ward-PV method is similar to the Ward-PV, except that the capacitive buses are preserved in the external area. The following equations illustrate the Ward-PV procedure. To preserve the external system $\mathrm{PV}$ buses, $Y_{Q Q}$ and $Y_{V V}$ are the admittance matrix of $P Q$ and $P V$ buses in the external zone, respectively.

$$
\begin{gathered}
{\left[\begin{array}{cccc}
Y_{Q Q} & Y_{Q V} & Y_{Q b} & 0 \\
Y_{V Q} & Y_{V V} & Y_{V b} & 0 \\
Y_{b Q} & Y_{b V} & Y_{b b}{ }^{e}+Y_{b b}{ }^{i} & Y_{b i} \\
0 & 0 & Y_{i b} & Y_{i i}
\end{array}\right]\left[\begin{array}{c}
E_{Q} \\
E_{V} \\
E_{b} \\
E_{i}
\end{array}\right]} \\
\quad=\left[\begin{array}{c}
I_{Q} \\
I_{V} \\
I_{b} \\
I_{i}
\end{array}\right] .
\end{gathered}
$$

To remove the external system $\mathrm{PQ}$ buses, voltage vector $E_{Q}$ must be removed from Eq. (13). In this 
regard, $E_{Q}$ is obtained from the first row of Eq. (13):

$$
\begin{aligned}
& Y_{Q Q} E_{Q}+Y_{Q V} E_{V}+Y_{Q b} E_{b}=I_{Q}, \\
& E_{Q}=Y_{Q Q}{ }^{-1}\left(I_{Q}-\left(Y_{Q b} E_{b}+Y_{Q V} E_{V}\right)\right) .
\end{aligned}
$$

Substituting $E_{Q}$ into the second and third rows of Eq. (15) would result in the following equations:

$$
\begin{aligned}
&\left(Y_{V b}\right.\left.-Y_{V Q} Y_{Q Q}{ }^{-1} Y_{Q b}\right) E_{b} \\
&+\left(Y_{V V}-Y_{V Q} Y_{Q Q}{ }^{-1} Y_{Q V}\right) E_{V}=I_{V} \\
&-\left(Y_{V Q} Y_{Q Q}{ }^{-1} I_{Q}\right) \\
&\left(Y_{b V}-Y_{b Q} Y_{Q Q}{ }^{-1} Y_{Q V}\right) E_{V}+\left(Y_{b b}{ }^{e}-Y_{b Q} Y_{Q Q}{ }^{-1} Y_{Q b}\right) \\
& E_{b}+Y_{b b}{ }^{i} E_{b}+Y_{b i} E_{i}=I_{b}-Y_{b Q} Y_{Q Q}{ }^{-1} I_{Q} \cdot
\end{aligned}
$$

According to Eqs. (16) and (17), we have:

$$
\begin{aligned}
& I_{e q V}=Y_{V Q} Y_{Q Q}{ }^{-1} I_{Q}, \\
& I_{e q b}=Y_{b Q} Y_{Q Q}{ }^{-1} I_{Q},
\end{aligned}
$$

where $I_{e q V}$ is the extra injection current in the external PV buses, and $I_{e q b}$ the extra injection current in the boundary buses. According to Eq. (13), $Y_{e q}$ is as follows:

$$
\begin{aligned}
Y_{e q}= & {\left[\begin{array}{cc}
Y_{V V} & Y_{V b} \\
Y_{b V} & Y_{b b}
\end{array}\right]-\left[\begin{array}{c}
Y_{V Q} \\
Y_{b Q}
\end{array}\right] \times\left[Y_{Q Q}\right]^{(-1)} } \\
& \times\left[\begin{array}{ll}
Y_{Q V} & Y_{Q b}
\end{array}\right] .
\end{aligned}
$$

The injection complex power in the boundary and external PV buses is obtained from the injection currents.

$$
\begin{aligned}
& S_{e q b}=E_{b}\left(Y_{b Q}\right)^{*}\left(Y_{Q Q}{ }^{*}\right)^{-1} E_{Q}{ }^{-1} S_{Q}, \\
& S_{e q V}=E_{V}\left(Y_{V Q}\right)^{*}\left(Y_{Q Q}{ }^{*}\right)^{-1} E_{Q}{ }^{-1} S_{Q},
\end{aligned}
$$

where $S_{e q b}$ and $S_{e q V}$ are the injection complex power in the boundary and PV buses, respectively.

\section{Procedure of proposed OCVC}

Followed by partitioning and reducing the dimensions of the power system, the method proposed in [3941] was employed to solve the OCVC problem. In these studies, OCVC was solved in two online and offline steps to boost the speed and adaptive feature. In [39], the system faults were first divided into two categories of anticipated and unanticipated faults. The former includes outage of a line or generator from the system, and the latter refers to any other fault in the system or a combination of several anticipated faults. Then, for each anticipated fault, the OCVC was solved in the offline mode and a set of non-dominated solutions was obtained and saved in the knowledge base of control system. Since there is no time limitation, the numbers of both initial population iterations of offline calculations are assumed to be 100 . In addition, the offline calculations were done in an unreduced system.

Whenever an anticipated fault occurs in the power system, the existing data relevant to this fault in the knowledge base is recalled to be applied to the system; however, due to the possibility of a change in the operating point of the system when the fault occurs in $[40,46]$, a local search system is introduced to update the set of solutions already available in the knowledge base. Therefore, the initial population of 10 cases is selected from the knowledge base. The initial population is chosen in a way as to ensure an acceptable diversity. To this end, the crowding distance concept is taken into account. The number of iterations of the local search is considered to be 10 . This method is adaptable to any operating point in the power system, hence called adaptive method.

In case an unanticipated fault occurs in the system, the location of the fault is determined in the first step, and the data relevant to this fault location is called from the knowledge base, thus forming a small search space $[40,41]$. Then, the online learning algorithm, introduced in [40,41], determines the appropriate solutions in this search space. In online learning, the numbers of iterations and initial population are both assumed to be 20. The flowchart of the proposed algorithm is presented in Figure 4.

\section{Simulation results}

To evaluate the performance of the proposed OCVC based on the modified Ward-PV, a New England 39 bus system was employed. This system contains 10 generators. The reference voltage of each generator is considered a control parameter varying from 0.9 to $1.1 \mathrm{pu}$. There are also 10 capacitors in Buses 3, 4, 7, $8,12,15,16,18,21$, and 26 changing from 0 to $0.3 \mathrm{pu}$ in Step 0.1. In addition, the system includes 21 load buses, hence 21 locations for load shedding. The load shedding step is assumed as $0.05 \mathrm{pu}$. Moreover, due to the OEL of each generator, the maximum excitation voltage of each generator is obtained as $2 \mathrm{pu}$. It should be mentioned that the values of the reference voltage of all generators and capacitors in their basic states are computed as 1 and $0 \mathrm{pu}$, respectively. It is assumed that all required parameters such as voltage, current, active and reactive power, and location of the fault are available. A core-i7 computer with a $1.6 \mathrm{GHz}$ processor was used for simulations. In the following, scenarios will be reviewed: 


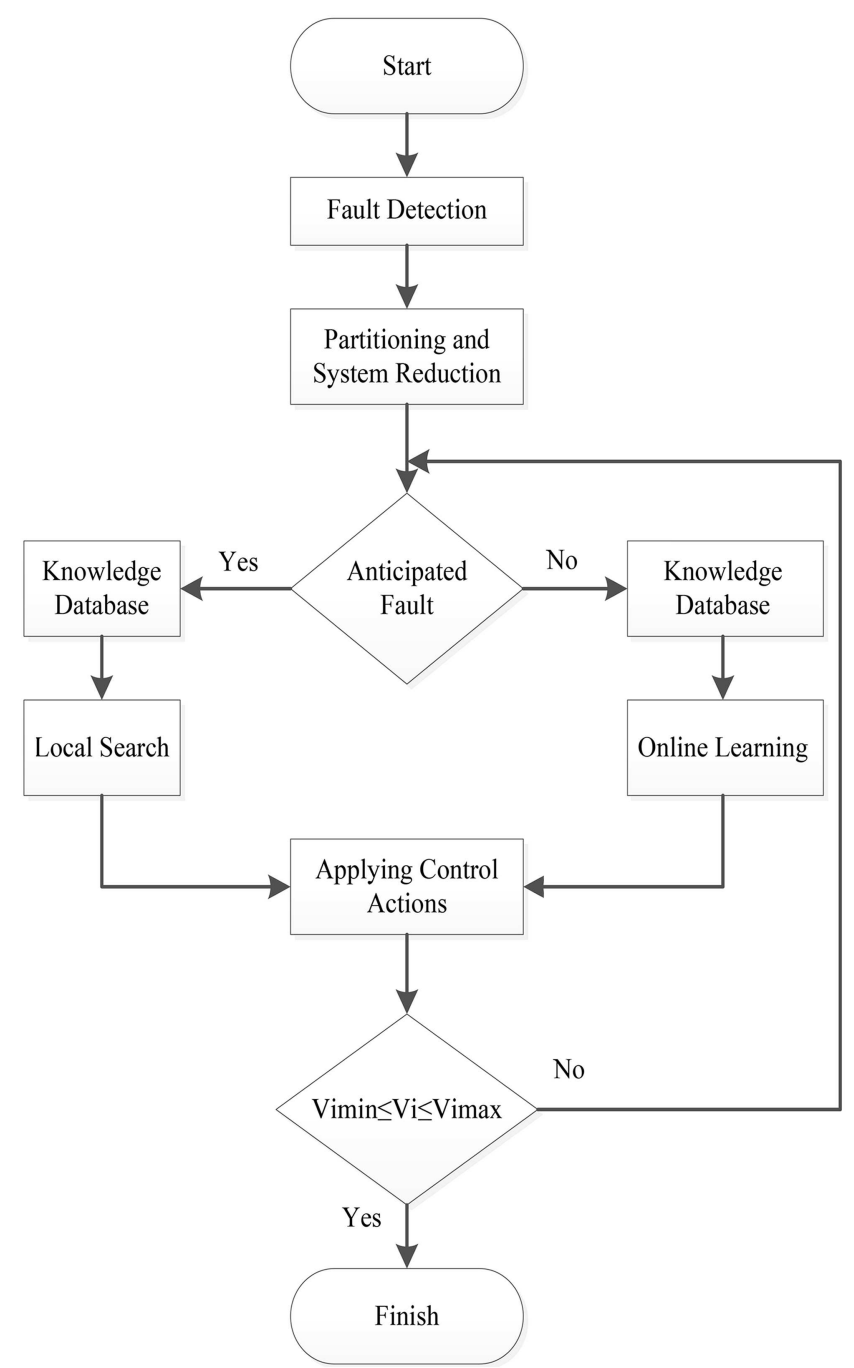

respectively. In addition, $N_{L}$ and $\Delta t$ are the number of buses and the time the control system takes to be executed, respectively.

(b) Investigation of the role system partitioning before and after the fault occurrence.

Scenario II: Simultaneous outage of lines 21-22 and $\% 20$ overload in bus 21 , which is an unanticipated fault, are taken into account to test the performance of the proposed method in case of unanticipated fault.

Scenario III: The objective of this scenario is to evaluate the performance of the proposed method against consecutive faults. Generator 32 and lines 6-7 tripped at two different times. This fault is considered an unanticipated fault.

The algorithm proposed to solve a multi objective optimization problem is jumping genes paradigm optimization algorithm [34-40]. In Table 4, the preference matrixes are represented from tracing reference voltage and economic consideration (minimization the number of control actions) to choose the best non-dominated solution and its application on the system. In all scenarios, it is assumed that the fault occurs at second 30 . The control actions will be in times $60 \mathrm{~s}, 90 \mathrm{~s}, 120 \mathrm{~s}$ and etc.

\subsection{Scenario I}

With outage of generator 32, as observed in Figure 5, the voltage of the power system was collapsed without any control system. It is an anticipated fault where

Figure 4. Proposed algorithm to solve the OCVC problem.

Scenario I: Anticipatable fault occurrence, outing generator 32 with two goals:

(a) Comparison of the methods based on dimension reduction: Modified Ward-PV and linearization method introduced in [40]. The following index is used to achieve this goal:

$$
C I=\frac{100}{\left(N_{L} \times \Delta t\right)} \sum_{\Delta t} \sum_{N_{L}}\left|V_{i, t}-V_{i, t}^{*} / V_{i, t}^{*}\right|
$$

where $V_{i, t}$ and $V_{i, t}^{*}$ are the voltages of bus $i$ at time $t$ in two reduced and unreduced systems,

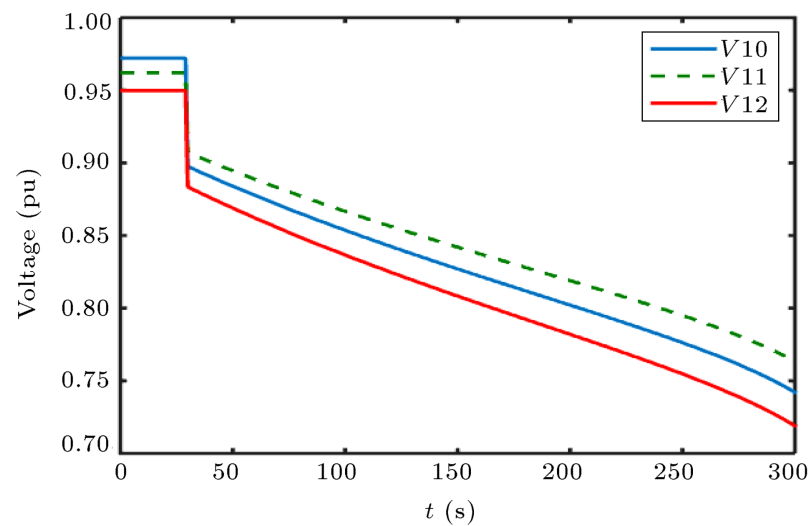

Figure 5. Performance of the system after outage of generator 32 without a control system.

Table 4. Preference matrix of tracing reference voltage and economic consideration.

\begin{tabular}{|c|c|c|c|c|c|c|c|}
\hline \multicolumn{4}{|c|}{ Tracing reference voltage } & \multicolumn{4}{|c|}{ Economic consideration } \\
\hline & $f_{1}=J_{\sum v i}$ & $f_{2}=J_{a c t}$ & $f_{3}=J_{\text {local }}$ & & $f_{1}=J_{\sum v i}$ & $f_{2}=J_{a c t}$ & $f_{3}=J_{\text {local }}$ \\
\hline$f_{1}=J_{\sum v i}$ & 1 & 5 & 3 & $f_{1}=J_{\sum v i}$ & 1 & $1 / 3$ & $1 / 2$ \\
\hline$f_{2}=J_{a c t}$ & $1 / 5$ & 1 & $3 / 5$ & $f_{2}=J_{a c t}$ & 3 & 1 & $3 / 2$ \\
\hline$f_{3}=J_{l o c a l}$ & $1 / 3$ & $5 / 3$ & 1 & $f_{3}=J_{l o c a l}$ & 2 & $2 / 3$ & 1 \\
\hline
\end{tabular}


the OCVC problem was formerly solved in an offline mode with the initial population of 100 and at iteration number 100. Of note, there is a set of non-dominated solutions in the knowledge base of this fault. The priority is to find optimal solutions with no load shedding. There are 14 sets of non-dominated solutions without load shedding in the knowledge base for outage of generator 32. That control system is given in Figure 6 according to which 10 superior solutions are chosen based on crowding distance concept from 14 possible solutions to form the initial population for local search. They are marked with "o".

To check the adaptability of the control method in this scenario, all loads randomly changed from 0.95 to 1.05 in the basic load.

Figure 7 depicts the partitioning of the power system into three subsystems before and after fault occurrence. Zone 1 where the fault occurred is regarded as the internal subsystem and the two other subsystems are the external subsystems. Followed by the outage of generator 32, if the power system is partitioned after the fault occurrence, generator 30 will be in the internal zone due to its role in voltage recovery. On the contrary, if the power system is partitioned before fault occurrence, generator 30 will be in the external zone. Therefore, it is better to do the partitioning after fault occurrence.

To precisely evaluate both modified Ward-PV and

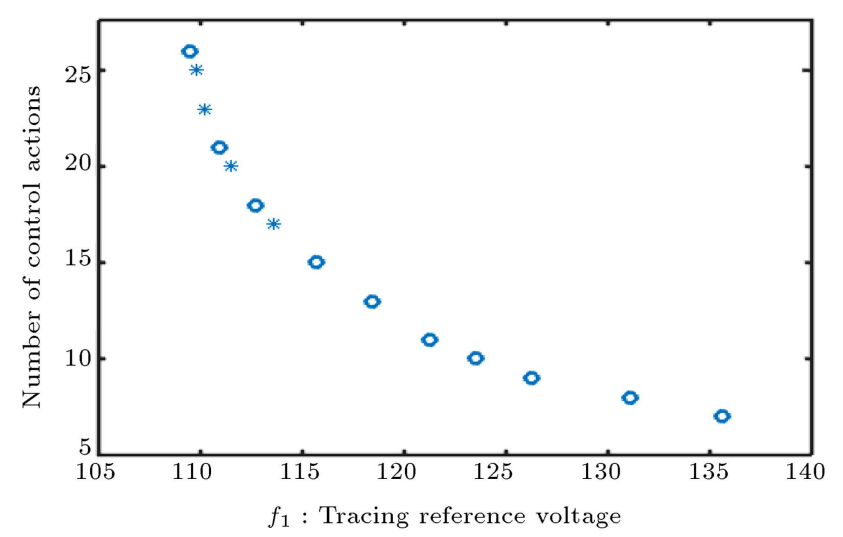

Figure 6. The set of non-dominated answers.

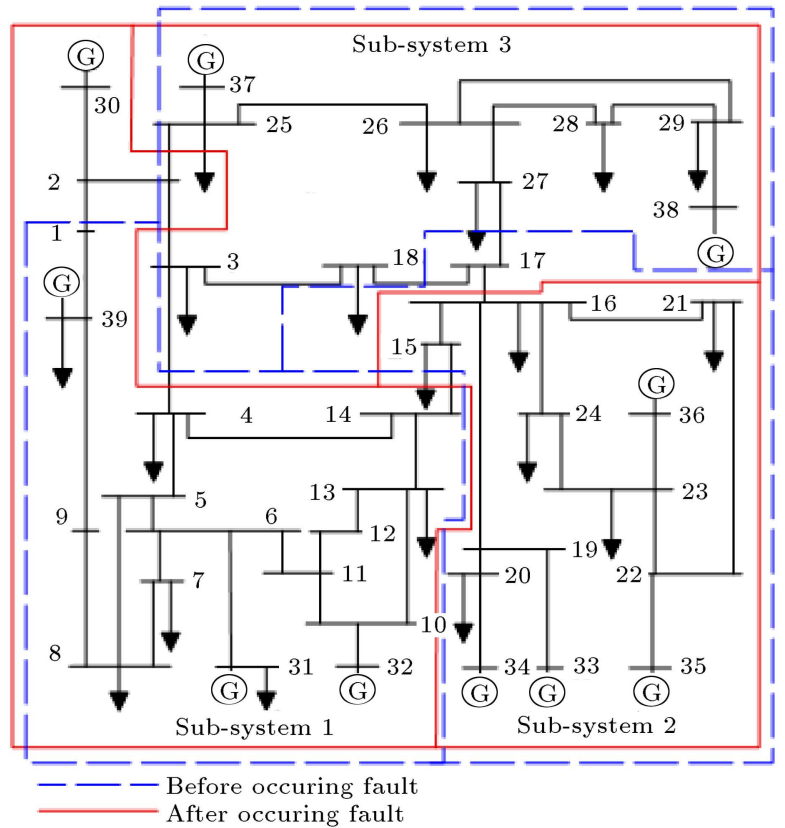

Figure 7. Partitioning of the power system for Scenario I.

linearization methods, it is assumed that the OCVC problem is solved using these methods and the control variables are applied to the unreduced system (the complete model of the system). Table 5 makes a comparison between the responses of both modified Ward-PV and linearization methods to the unreduced model. It can be concluded that the modified Ward$\mathrm{PV}$ is twice more precise than the linearization method; however, the speed of the former is lower than that of the latter. The time needed for searching in the unreduced system is about 16 seconds, but as observed in Table 5, the same time is be reduced to 8.5 and 11.4 seconds in the linearization and modified WardPV methods, respectively, mainly due to the number of equations (see Table 6 ).

Figures 8 and 9 show the voltage of bus 11 in the presence of the introduced control systems with two objective functions of tracing reference voltage and minimizing control actions. The following results can be obtained from this scenario:

1. Adaptability of both linearization and modified

Table 5. A comparison of the performance of both linearization and modified Ward-PV methods.

\begin{tabular}{ccccccccc}
\hline & $\begin{array}{c}\text { Time for } \\
\text { each local } \\
\text { search }(\mathbf{s})\end{array}$ & \multicolumn{3}{c}{$\begin{array}{c}\text { Tracing reference } \\
\text { voltage }\end{array}$} & \multicolumn{3}{c}{$\begin{array}{c}\text { Number of control } \\
\text { actions }\end{array}$} \\
\cline { 3 - 8 } & & CI & $\boldsymbol{f}_{\mathbf{2}}$ & $\boldsymbol{f}_{\mathbf{3}}$ & CI & $\boldsymbol{f}_{\mathbf{2}}$ & $\boldsymbol{f}_{\mathbf{3}}$ \\
\hline $\begin{array}{c}\text { Reduced based on } \\
\text { linearization method }\end{array}$ & 8.5 & 1.98 & 24 & 0 & 1.73 & 11 & 0 \\
$\begin{array}{c}\text { Reduced based on } \\
\text { modified Ward-PV method }\end{array}$ & 11.4 & 0.91 & 23 & 0 & 0.84 & 11 & 0 \\
\hline
\end{tabular}


Table 6. Number of system equations in each method.

\begin{tabular}{ccc}
\hline Method & $\begin{array}{c}\text { Number of equations } \\
\text { after fault } \\
\text { occurrence }\end{array}$ & $\begin{array}{c}\text { Number of equations } \\
\text { before fault } \\
\text { occurrence }\end{array}$ \\
\hline Non-reduced model & 190 & 190 \\
Reduced based on linearization method & 79 & 66 \\
Reduced based on modified Ward-PV method & 139 & 137 \\
\hline
\end{tabular}

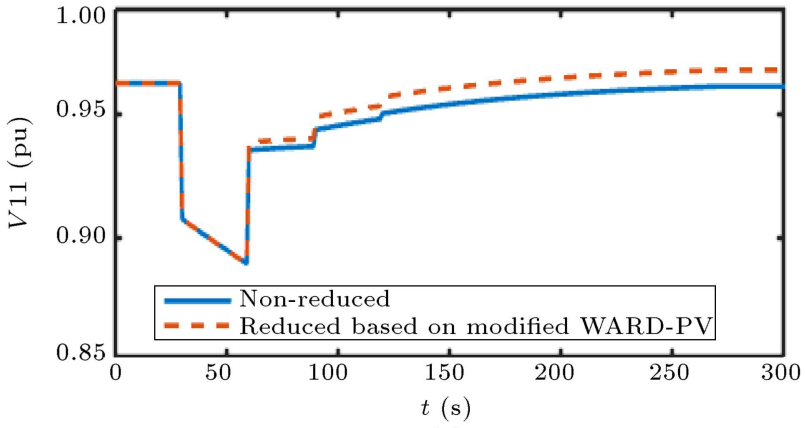

(a)

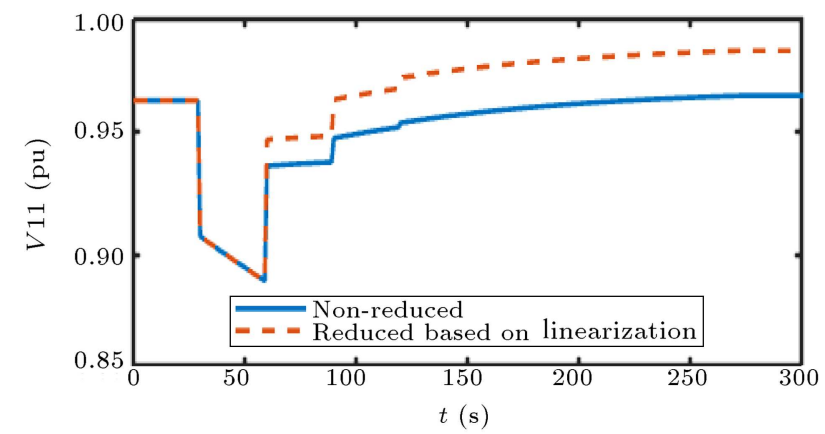

(b)

Figure 8. Performance of the control system with a reference voltage tracking approach.

Ward-PV methods owing to the alteration of the operating point;

2. Higher speed of linearization method than that of the modified Ward-PV, which would, indeed, lead to less accuracy;

3. Respect for customer's right (the numbers of load shedding in both methods for objective functions of tracing reference voltage and minimizing control actions are equal to zero);

4. Flexibility of both methods in solving the multiobjective optimization problem.

\subsection{Scenario II}

In this scenario, Lines $21-22$ are outed and simultaneously, there is an overload of $20 \%$ in Bus 21. Due to this fault, there will be a voltage collapse in the absence of any control system, as depicted in Figure 10. Figure 11 represents the system partitioning after the fault occurrence, which is an unanticipated fault.

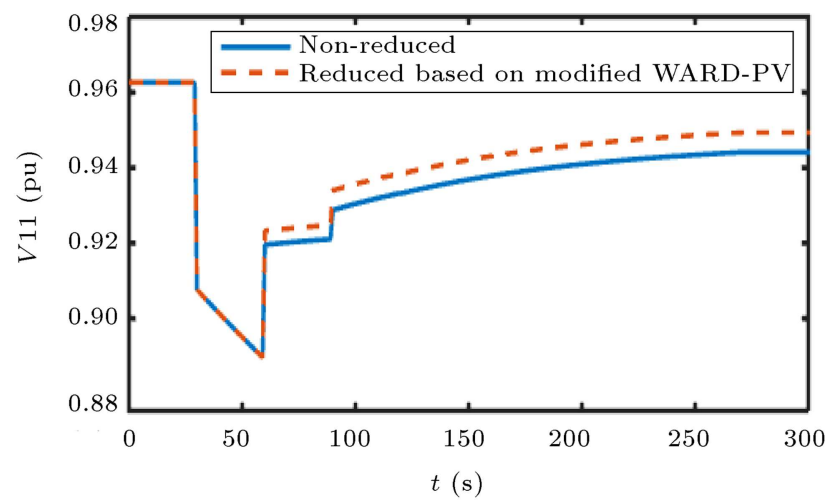

(a)

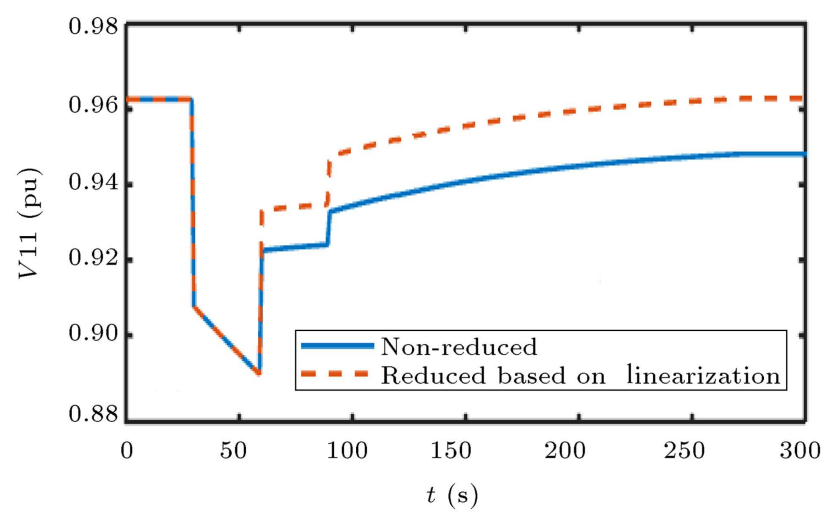

(b)

Figure 9. Performance of the control system with reducing the control actions.

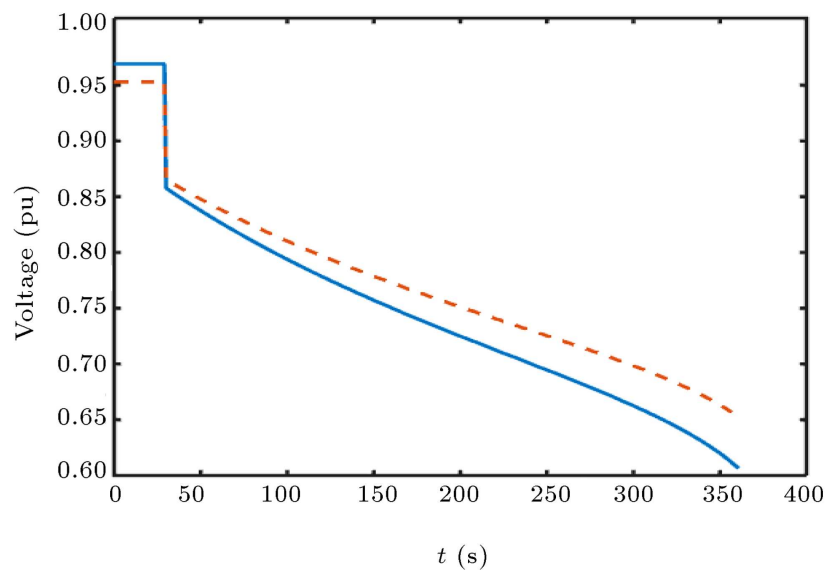

Figure 10. Performance of system in Scenario II, without a control system. 


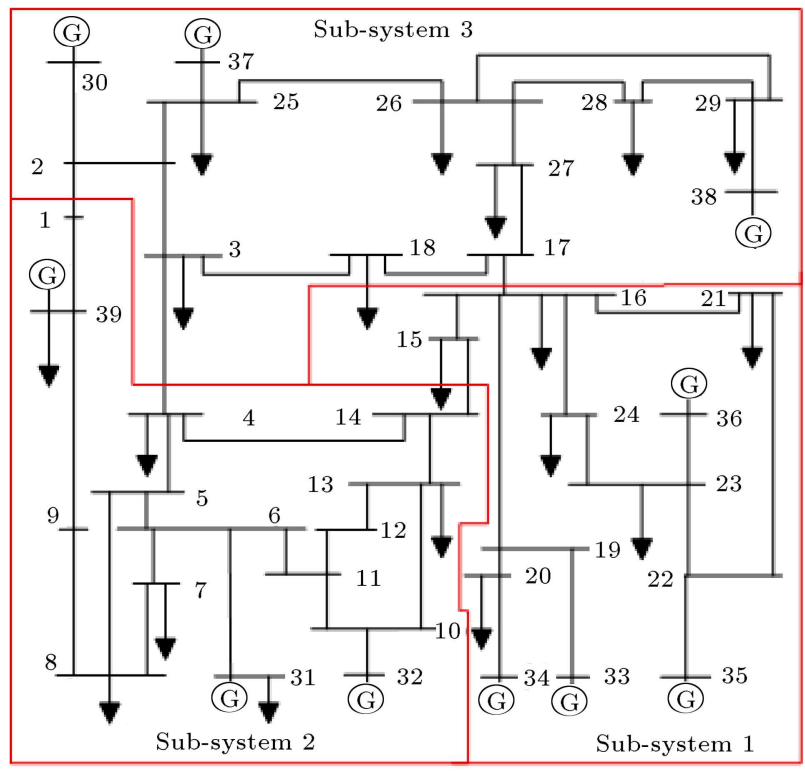

Figure 11. System partitioning after fault in Scenario II.

As a result of this fault occurrence, all data of buses 21 and 22 saved in the knowledge base were called to form the initial population of online learning. Tables 7 and 8 make a thorough comparison of the results obtained from both two modified Ward-PV and linearization methods. In this scenario, the accuracy of the modified Ward-PV method is better than that of the linearization method. However, the speed of the linearization method is higher than that of the modified Ward-PV.

In Figures 12 and 13, the voltage of bus 21 is given by tracing reference voltage and minimizing the number of control actions.

\subsection{Scenario III}

To evaluate the performance of the proposed method versus the consecutive faults, another scenario with two

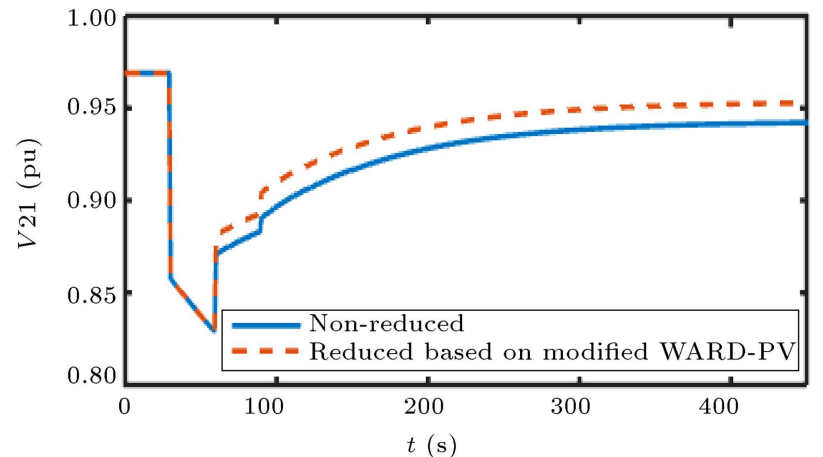

(a)

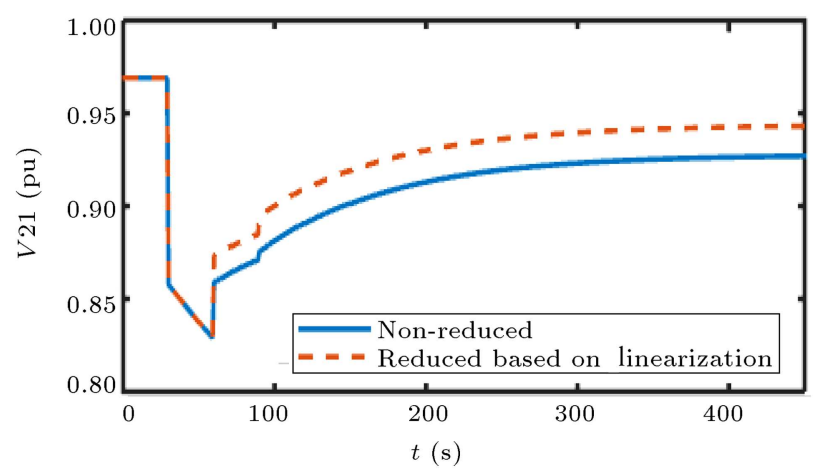

(b)

Figure 12. Performance of the control system through reference voltage tracking approach in Scenario II.

different simulations was taken into account. In both cases, the first and second faults were regarded as the outages of generator 32 and lines $6-7$, respectively. The first fault occurred at $t=30 \mathrm{~s}$ for both simulations, while the second one occurred at $75 \mathrm{~s}$ and $105 \mathrm{~s}$. Since the second fault occurred a short time after the first fault, these two faults were considered to be combined and unanticipated faults. Figure 14 evaluates the performance of the control system against these

Table 7. Comparison of the performance of both linearization and modified Ward-PV methods in Scenario II.

\begin{tabular}{|c|c|c|c|c|c|c|c|}
\hline \multirow[b]{2}{*}{ Method } & \multirow[t]{2}{*}{$\begin{array}{l}\text { Time for } \\
\text { each local } \\
\text { search (s) }\end{array}$} & \multicolumn{3}{|c|}{$\begin{array}{c}\text { Tracing reference } \\
\text { voltage }\end{array}$} & \multicolumn{3}{|c|}{$\begin{array}{c}\text { Number of control } \\
\text { actions }\end{array}$} \\
\hline & & CI & $f_{2}$ & $f_{3}$ & CI & $f_{2}$ & $f_{3}$ \\
\hline Reduced based on linearization method & 8.2 & 3.1 & 31 & 3 & 1.93 & 14 & 2 \\
\hline Reduced based on modified Ward-PV method & 11.2 & 1.3 & 29 & 4 & 0.9 & 13 & 2 \\
\hline
\end{tabular}

Table 8. Number of system equations in each method based on Scenario II.

\begin{tabular}{cc}
\hline Method & $\begin{array}{c}\text { Number of equations } \\
\text { after fault } \\
\text { occurrence }\end{array}$ \\
\hline Non-reduced model & 190 \\
Reduced based on linearization method & 70 \\
Reduced based on modified Ward-PV method & 136 \\
\hline
\end{tabular}




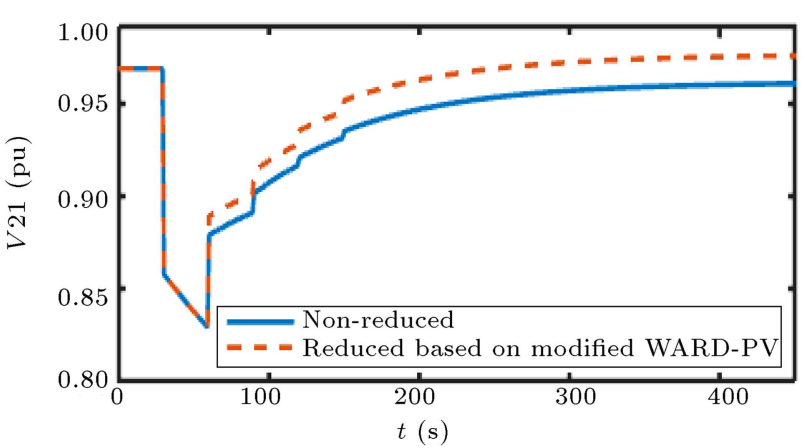

(a)

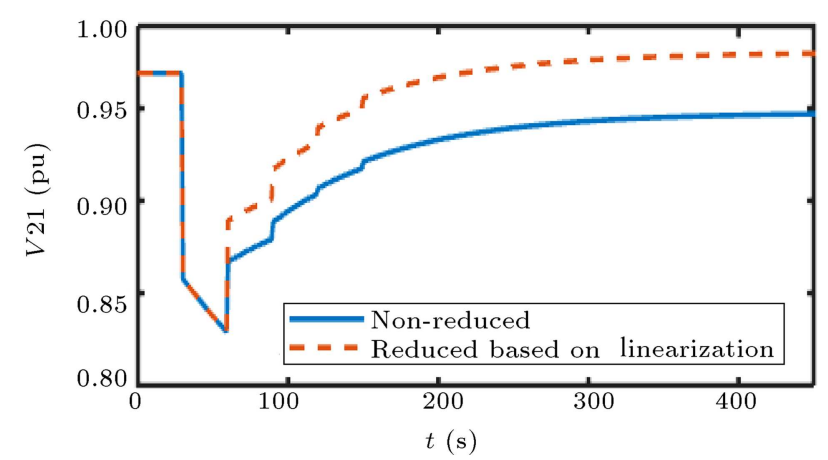

(b)

Figure 13. Performance of the control system through reducing the control actions in Scenario II.

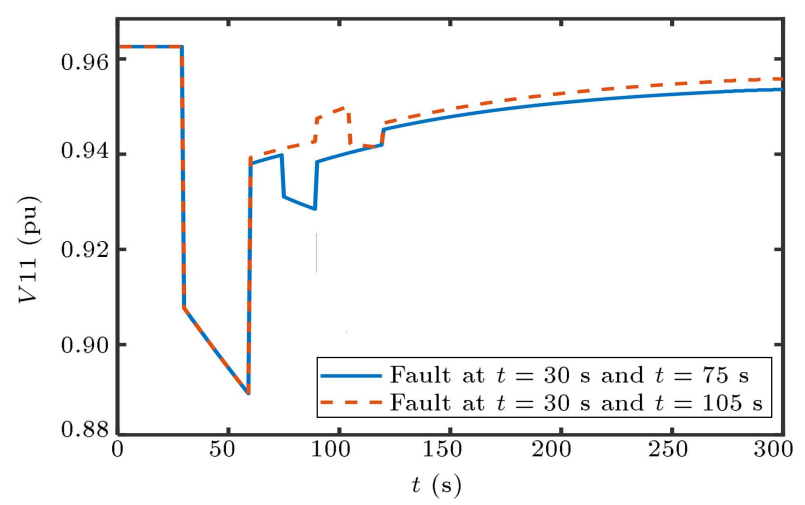

Figure 14. Performance of the control system against consecutive faults in Scenario III.

Table 9. The number of control actions and load shedding steps in Scenario III.

\begin{tabular}{ccc}
\hline Method & $\boldsymbol{f}_{\mathbf{2}}$ & $\boldsymbol{f}_{\mathbf{3}}$ \\
\hline Faults at $t=30 \mathrm{~s}$ and $75 \mathrm{~s}$ & 25 & 3 \\
Faults at $t=30 \mathrm{~s}$ and $105 \mathrm{~s}$ & 24 & 4 \\
\hline
\end{tabular}

consecutive faults, considering the tracing reference voltage. Table 9 presets the number of control actions and load shedding steps.

\section{Conclusion}

The current research aimed to establish a quick method for solving the OCVC problem based on reducing the dimensions of the power system using the modified Ward-PV. To this end, followed by fault occurrence, the power system was partitioned to reduce the dimensions of the system based on minimizing the reactive power flow from the lines between the subsystems. After partitioning, the zone where the fault occurred was considered as the internal subsystem. Since the voltage instability phenomenon is only a local issue in early stages of fault occurrence in the power system, the external area can be replaced by the equivalent model. Therefore, the modified Ward-PV was used to reduce the size of the power system while maintaining the performance accuracy. The simulations results obtained from New England IEEE 39-Bus System revealed that the proposed method was adaptive, flexible, and applicable to large power systems. In addition, it outperformed the linearization method in terms of precision. However, low speed in problem-solving is its major drawback.

\section{Abbreviations \\ OCVC Optimal Coordinated Voltage Control \\ MPC Model Predictive Control \\ CVC Coordinated Voltage Control \\ PID Proportional Integral Derivative \\ PSO Particle Swarm Optimization \\ EMS Energy Management System \\ OLTC On-Load Tap Changers \\ MCDM Multi-Criteria Decision-Making}

\section{References}

1. Kundur, P., Paserba, J., Ajjarapu, V., et al. "Definition and classification of power system stability IEEE/CIGRE joint task force on stability terms and definitions", Int. J. IEEE Transactions on Power Systems., 19(3), pp. 1387-1401 (2004).

2. Rabiee, A., Mohseni-Bonab, S.M., Parniani, M., et al. "Optimal cost of voltage security control using voltage dependent load models in presence of demand response", Int. J. IEEE Transactions on Smart Grid., 10(3), pp. 2383-2395 (2018).

3. Taylor, C.W. and Erickson, D.C. "Recording and analyzing the July 2 cascading outage [Western USA power system]", Int. J. IEEE Computer Applications in Power, 10(1), pp. 26-30 (1997).

4. Ye, X., Le, J., Liu, Y., et al. "A coordinated consistency voltage stability control method of active distribution grid", Int. J. of Modern Power Systems and Clean Energy, 6(1), pp. 85-94 (2018).

5. Esaka, T., Kataoka, Y., Ohtaka, T., et al. "Voltage stability preventive and emergency preventive control using VIPIt sensitivities", Int. Conf. IEEE PES Power Systems Conference and Exposition., New York, USA, pp. 509-516 (2004). 
6. Voropai, N.I., Kurbatsky, V.G., Tomin, N.V., et al. "Preventive and emergency control of intelligent power systems", 3th Int. Conf. IEEE PES Innovative Smart Grid Technologies Europe (ISGT Europe), Berlin, Germany (2012).

7. Antoniadou-Plytaria, K.E., Kouveliotis-Lysikatos, I.N., Georgilakis, P.S., et al. "Distributed and decentralized voltage control of smart distribution networks: Models, methods, and future research", Int. J. IEEE Transactions on Smart Grid., 8(6), pp. 2999-3008 (2017).

8. Vournas, C. and Karystianos, M. "Load tap changers in emergency and preventive voltage stability control", Int. J. IEEE Transactions on Power Systems, 19(1), pp. 492-498 (2004).

9. Gu, M., Meegahapola, L.G., and Wong, A.K. "Coordinated voltage and frequency control in hybrid AC/MTHVDC power grids for stability improvement", Int. J. IEEE Transactions on Power Systems, 36(1), pp. 635347 (2021).

10. Corsi, S., Pozzi, M., Sabelli, C., et al. "The coordinated automatic voltage control of the Italian transmission Grid-Part I: Reasons of the choice and overview of the consolidated hierarchical system", Int. J. IEEE Transactions on Power Systems, 19(4), pp. 1723-1732 (2004).

11. Corsi, S., Pozzi, M., Sforna, M., et al. "The coordinated automatic voltage control of the Italian transmission grid-Part II: control apparatuses and field performance of the consolidated hierarchical system Reasons of the choice and overview of the consolidated hierarchical system", Int. J. IEEE Transactions on Power Systems, 19(4), pp. 1733-1741 (2004).

12. Geidl, M. "Implementation of coordinated voltage control for the Swiss transmission system", 15th Int. Conf. IEEE Mediterranean Electrotechnical Conference., Valletta, Malta (2010).

13. Van Hecke, J., Janssens, N., Deuse, J., et al. "Coordinated voltage control experience in Belgium", In Coordinated Voltage Control in Transmisson Network, p. CIGRE, pp. 24-25 (2007).

14. Paul, J., Leost, J., and Tesseron, J. "Survey of the secondary voltage control in France: Present realization and investigations", Int. J. IEEE Transaction on Power Systems, 2(2), pp. 505-511 (1987).

15. Beccuti, A.G., Demiray, T.H., Andersson, G., et al. "A Lagrangian decomposition algorithm for optimal emergency voltage control", Int. J. IEEE Transactions on Power Systems, 25(4), pp. 1769-1779 (2010).

16. Wang, X., Wang, C., Xu, T., et al. "Optimal voltage regulation for distribution networks with multimicrogrids", Int. J. ScienceDirect. Applied Energy, 210, pp. 1027-1036 (2018).

17. Granada, M., Rider, M.J., Mantovani, J., et al. "A decentralized approach for optimal reactive power dispatch using a Lagrangian decomposition method", Int. J. ScienceDirect. Electric Power systems Research, 89, pp. 148-156 (2012).
18. Islam, S.R., Muttaqi, K.M., and Sutanto, D. "Multiagent receding horizon control with neighbour-toneighbour communication for prevention of voltage collapse in a multi-area power system", In. J. IET Generation, Transmission \& Distribution, 8(9), pp. 1604-1615 (2014).

19. Li, P., Zhang, B., Cheng, C., et al. "A distributed model prediction based method for coordinated voltage control of power system", 10th IET. Int. Conf. on Developments in Power System Protection (DPSP 2010), Managing the Change (2010).

20. Van Pham, H. and Ahmed, S.N. "Multi-agent based approach for intelligent control of reactive power injection in transmission systems", In Dynamic Vulnerability Assessment and Intelligent Control: For Sustainable Power System, P. Wiley-IEEE Press, First Ed., p. 269 (2018).

21. Liu, S., Li, Y., Cao, Y., et al. "Distributed model predictive control algorithms for emergency voltage stability control of power systems", Int. Conf. IEEE PES Asia-Pacific Power and Energy Engineering Conference (APPEEC), Xi'an, China, pp. 2597-2600 (2016).

22. Vallem, M.R., Vyakaranam, B., Holzer, J.T., et al. "Power system decomposition for practical implementation of bulk-grid voltage control methods", 19th Int. Conf. on Intelligent System Application to Power Systems (ISAP), San Antonio, TX, USA, pp. 1-6 (2017).

23. Wang, S., Liu, M., Hu, B., et al. "Emergency voltage control based on MPC, Radau collocation and moving finite elements technique", Int. Conf. on Power System Technology., Zhejiang, China, pp. 1-7 (2010).

24. Larsson, M. and Karlsson, D. "Coordinated system protection scheme against voltage collapse using heuristic search and predictive control", Int. J. IEEE Power Engineering Review., 22(6), pp. 59-59 (2002).

25. Wen, J., Wu, Q., Turner, D., et al. "Optimal coordinated voltage control for power system voltage stability", Int. J. IEEE Transactions on Power Systems., 19(2), pp. 1115-1122 (2004).

26. Larsson, M. "A model-predictive approach to emergency voltage control in electrical power systems", 43th IEEE. Conf. on Decision and Control (CDC)., Nassau, Bahamas (2004).

27. Sun, X. and Zhang, W. "Coordinated optimal voltage control strategy in distribution networks with multi-microgrids", IEEE. Conf. IEEE PES AsiaPacific Power and Energy Engineering Conference (APPEEC)., Kota Kinabalu, Malaysia, pp. 88-93 (2018).

28. Jin, Li., Kumar, R., and Elia, N. "Security constrained emergency voltage stabilization: A Model Predictive Control based approach", 47th IEEE. Conf .on Decision and Control., Cancun, Mexico, pp. 2469-2474 (2008).

29. Li, Y., Hill, D., and Wu, T. "Nonlinear model predictive control with immune optimization for voltage 
security control", 5th Int. Conf. Intelligent Control and Automation., Hangzhou, China, pp. 5189-5193 (2004).

30. Zhang, F., Chan, K.W., and Fang, D.Z. "Optimal coordinated voltage emergency control against voltage collapse", Int. J. of Electrical Power \& Energy Systems., 53, pp. 442-449 (2013).

31. Otomega, B., Glavic, M., and Van Cutsem, T. "A two-level emergency control scheme against power system voltage instability", Int. J. Control Engineering Practice., 30, pp. 93-104 (2014).

32. Negenborn, R., Leirens, S., De Schutter, B., et al. "Supervisory nonlinear MPC for emergency voltage control using pattern search", Int. J. Control Engineering Practice., 7, pp. 841-848 (2009).

33. Larsson, M., Hill, D.J., and Olsson, G. "Emergency voltage control using search and predictive control", Int. J. of Electrical Power \& Energy Systems, 24(2), pp. 121-130 (2002).

34. Amraee, T., Ranjbar, A., and Feuillet, R. "Adaptive under-voltage load shedding scheme using model predictive control", Int. J. of Electric Power Systems Research, 81(7), pp. 1507-1513 (2011).

35. Pourjafari, E. and Mojallali, H. "Predictive control for voltage collapse avoidance using a modified discrete multi-valued PSO algorithm", Int. J. ISA Transactions, 50(2), pp. 195-200 (2011).

36. Ma, H., Man, K., and Hill, D.J. "A jumping genes scheme for multi-objective coordinated voltage control", 4th Int. Conf. on Industrial Informatics, Singapore (2006).

37. Ma, H.M., Ng, K.T., and Man K.F. "Multiobjective coordinated power voltage control using jumping genes paradigm", Int. J. IEEE Transactions on Industrial Electronics, 55(11), pp. 4075-4084 (2008).

38. Ma, H.M., Ng, K.T., and Man K.F. "A multiple criteria decision-making knowledge-based scheme for realtime power voltage control", Int. J. IEEE Transactions on Industrial Informatics, 4(1), pp. 58-66 (2008).

39. Ma, H. and Hill, D.J. "Adaptive coordinated voltage control-Part I: Basic scheme", Int. J. IEEE Transactions on Power Systems, 29(4), pp. 1546-1553 (2013).

40. Ma, H. and Hill, D.J. "Adaptive coordinated voltage control-Part II: Use of learning for rapid response", Int. J. IEEE Transactions on Power Systems, 29(4), pp. 1554-1561 (2014).

41. Karbalaei, F. and Shahbazi, H. "A quick method to solve the optimal coordinated voltage control problem based on reduction of system dimensions", Int. J. Electric Power Systems Research, 142, pp. 310-319 (2017).

42. Islam, S.R., Muttaqi, K.M., and Sutanto, D. "A decentralized multiagent-based voltage control for catastrophic disturbances in a power system", Int. Conf. IEEE Industry Applications Society Annual Meeting., Lake Buena Vista, FL, USA (2013).
43. Zhao, B., Xu, Z., Xu, C., et al. "Network partitionbased zonal voltage control for distribution networks with distributed PV systems", Int. J. IEEE Transactions on Smart Grid., 9(5), pp. 4087-4098 (2018).

44. Islam, S.R., Sutanto, D., and Muttaqi, K.M. "Coordinated decentralized emergency voltage and reactive power control to prevent long-term voltage instability in a power system", Int. J. IEEE Transaction on Power Systems, 30(5), pp. 2591-2603 (2014).

45. Shahbazi, H. and Karbalaei, F. "Decentralized voltage control of power systems using multi-agent systems", Int. J. of Modern Power Systems and Clean Energy., 8(2), pp. 249-259 (2020).

46. Ma, H. and Hill, D.J. "A fast local search scheme for adaptive coordinated voltage control", Int. J. IEEE Transaction on Power Systems, 33(3), pp. 2321-2330 (2018).

47. Huang, Q., Huang, R., Hao, W., et al. "Adaptive power system emergency control using deep reinforcement learning", Int. J. IEEE Transactions on Smart Grid., 11(2), pp. 1171-1182 (2019).

48. Liu, H., Su, J., Qi, J., et al. "Decentralized voltage and power control of multi-machine power systems with global asymptotic stability", In. J. IEEE Access., 7, pp. 14273-14282 (2019).

49. Guzman, R., De Vicuna, L.G., Camacho, A., et al. "Receding-horizon model-predictive control for a three-phase VSI with an LCL filter", Int. J. IEEE Transactions on Industrial Electronics, 66(9), pp. 6671-6680 (2018).

50. Jin, T., Shen, X., Su, T., et al. "Model predictive voltage control based on finite control set with computation time delay compensation for PV systems", Int. J. IEEE Transactions on Energy Conversion, 34(1), pp. $330-338$ (2018).

51. Wu, F.F. and Monticelli, A. "Critical review of external network modelling for online security analysis", Int. J. of Electrical Power \& Energy Systems., 5(4), pp. 222-235 (1983).

52. Mehrjerdi, H., Lefebvre, S., Asber, D., et al. "Graph partitioning of power network for emergency voltage control", 9th Int. Conf. Asian Control Conference (ASCC)., Istanbul, Turkey, pp. 23-26 (2013).

53. Alzaareer, K., Saad, M., Mehrjerdi, H., et al. "Development of new identification method for global group of controls for online coordinated voltage control in active distribution networks", Int. J. IEEE Transactions on Smart Grid., 11(5), pp. 3921-3931 (2020).

54. Hosseinnezhad, V., Rafiee, M., Ahmadian, M., et al. "Optimal island partitioning of smart distribution systems to improve system restoration under emergency conditions", Int. J. of Electrical Power \& Energy Systems., 97, pp. 155-164 (2018).

\section{Biographies}

Mandana Hojati Tabatabaei received her BSc degree in Electronic Engineering from Islamic Azad 
University Garmsar Branch in 2008 and MSc degree in Power Electrical Engineering from Imam Khomeini International University of Qazvin in 2012. She is currently a PhD student in Power Electrical from Islamic Azad University-South Tehran Branch.

Hassan Siahkali (S'06) was born in Tehran, Iran in 1970. He received his BSc degree in Electronic Engineering from the Tabriz University, Tabriz, Iran in 1993 and MSc and $\mathrm{PhD}$ degrees both in Electrical Engineering from the Amir Kabir University of Technology and Sharif University of Technology, Tehran, Iran in 1997 and 2010, respectively. He worked as a project manager at the Niroo Research Institute (NRI) from 1999 to 2005. He has also been cooperating with Islamic Azad University-South Tehran Branch as an Assistant Professor since 2006. His main research interests are the electric power systems planning and operation and restructuring of power system.
Javad Olamaei (SM'18) received his BSc, MSc, and $\mathrm{PhD}$ degrees in Electrical Engineering in 1988, 1992, and 2008 from Tabriz University, Tabriz, Iran, Amir Kabir University of Technology (AUT), Tehran, Iran, and Islamic Azad University-Science and Research Branch, Tehran, Iran, respectively. He holds the Lecturer position from January 1997 to June 2009, the Assistant Professor position from June 2009 to February 2018, and Associate Professor position at Islamic Azad University-South Tehran Branch since February 2018. He was the head manager of Islamic Azad University South Tehran Branch from 2018 to 2019. From 2019 until now, he is the Deputy Minister of Science, Engineering and Agriculture of Islamic Azad University. He is the author of more than 100 international journal and conference papers. His teaching and research interests include power distribution systems, distribution automation system, micro grids, renewable energy, and VAR planning. 